\title{
LV-OP-2-5
}

\section{Liver transplantation for combined hepatocellular carcinoma and cholangiocarcinoma in Korea}

\author{
Jong Man $\mathrm{KIM}^{*, 1}$, Dong Jin $\mathrm{JOO}^{2}$, Shin $\mathrm{HWANG}^{3}$, Nam-Joon $\mathrm{YI}^{4}$, Je Ho RYU ${ }^{5}$, Yang Won NA ${ }^{6}$, Dong-Sik KIM, \\ Doo Jin $\mathrm{KIM}^{8}$, Young Kyoung $\mathrm{YOO}^{9}$, Hee Chul $\mathrm{YU}^{10}$, Myoung Soo $\mathrm{KIM}^{2}$ \\ 'Department of Surgery, Samsung Medical Center, Sungkyunkwan University School of Medicine, Seoul, Korea \\ ${ }^{2}$ Department of Surgery, Yonsei University College of Medicine, Seoul, Korea \\ ${ }^{3}$ Department of Surgery, Asan Medical Center, University of Ulsan College of Medicine, Seoul, Korea \\ ${ }^{4}$ Department of Surgery, Seoul National University College of Medicine, Seoul, Korea \\ ${ }^{5}$ Department of Surgery, Pusan National University College of Medicine, Yangsan, Korea \\ ${ }^{6}$ Department of Surgery, Ulsan University Hospital, University of Ulsan College of Medicine, Ulsan, Korea \\ ${ }^{7}$ Department of Surgery, Korea University College of Medicine, Seoul, Korea \\ ${ }^{8}$ Department of Surgery, Gachon University Gil Medical Center, Gachon University College of Medicine, Incheon, Korea \\ ${ }^{9}$ Department of Surgery, College of Medicine, The Catholic University of Korea, Seoul, Korea \\ ${ }^{10}$ Department of Surgery, Jeonbuk National University Medical School, Jeonju, Korea
}

Introduction: There is still no consensus about the actual role of liver transplantation (LT) in the therapeutic algorithm of combined hepatocellular-cholangiocarcinoma (cHCC-CC) because of low incidence and few clinical information.

Methods: We retrospectively identified 111 patients at nine centers from 2001 to 2019 in Korea.

Results: Of the 111 patients, $85.6 \%(\mathrm{n}=95)$ was male and the median age was 54 years (range, $31-66$ years). HBV is $82 \%(\mathrm{n}=91)$ and HCC is $96 \%$ (86.3\%) preoperatively. Seventy-four patients (66.7\%) received locoregional therapy (LRT) before LT. Median tumor size was $2.5 \mathrm{~cm}$ (range, $0.5-7.2 \mathrm{~cm}$ ) and the ratio of beyond Milan criteria was $40.5 \%(\mathrm{n}=45)$. The 1-year, 3-year, and 5-year disease-free survival rates and tumor-related patient survival rates were $77.6 \%, 56.3 \%$, and $51.1 \%$ and $84.4 \%, 63.8 \%$, and $56.7 \%$, respectively. The extrahepatic site was $75.5 \%$ as an initial tumor recurrence site in recurrent tumor patients. Numbers of LRT before LT $>3$ and tumor size $>3 \mathrm{~cm}$ were closely associated tumor recurrence and early tumor recurrence within 1 year after LT. Tumor size $>3 \mathrm{~cm}$ was only predisposing factor for tumor-related death.

Conclusions: It is difficult to diagnose cHCC-CC before LT, but a good prognosis can be expected if the tumor size is less than $3 \mathrm{~cm}$ in pathology. 